\title{
Alterations of Gut Microbiota in Patients With Intestinal Tuberculosis That Different From Crohn's Disease
}

\author{
Cong He ${ }^{1 \dagger}$, Huan Wang ${ }^{1 \dagger}$, Chen Yut ${ }^{2 \dagger}$, Chao Peng ${ }^{1}, X_{u}$ Shu $^{1}$, Wangdi Liao ${ }^{1 \neq}$ and \\ Zhenhua Zhu ${ }^{1 * \neq}$ \\ ${ }^{1}$ Department of Gastroenterology, The First Affiliated Hospital of Nanchang University, Nanchang, China, ${ }^{2}$ Department \\ of Radiology, The First Affiliated Hospital of Nanchang University, Nanchang, China
}

OPEN ACCESS

Edited by:

Bo Xiao,

Southwest University, China

Reviewed by:

Youlian Zhou,

Guangzhou First People's Hospital,

China

Zhan Zhang,

Emory Vaccine Centre, Yerkes National Primate Research Centre,

Emory University, United States

${ }^{*}$ Correspondence:

Zhenhua Zhu

zhuzhenhua19820122@163.com

tThese authors share first authorship

*These authors share last authorship

Specialty section: This article was submitted to

Nanobiotechnology,

a section of the journal

Frontiers in Bioengineering and

Biotechnology

Received: 28 February 2021 Accepted: 11 June 2021

Published: 06 July 2021

Citation:

He C, Wang H, Yu C, Peng C,

Shu X, Liao W and Zhu Z (2021)

Alterations of Gut Microbiota in Patients With Intestinal Tuberculosis That Different From Crohn's Disease. Front. Bioeng. Biotechnol. 9:673691. doi: 10.3389/fbioe.2021.673691
Intestinal tuberculosis (ITB) and Crohn's disease (CD) are chronic inflammatory bowel disorders that are associated with dysregulated mucosal immunity. The gut microbiota plays an important role in the regulation of host immunity and inflammatory response. Although mounting evidence has linked CD with the dysbiosis of gut microbiota, the characteristic profiles of mucosal bacteria in ITB remain unclear. The aim of this study was to assess the alterations of the gut microbiota in ITB and compare the microbial structure of ITB with CD. A total of 71 mucosal samples were collected from patients with ITB, $C D$, and healthy controls $(\mathrm{HC})$, and then, 16S rRNA gene sequencing was performed. The overall composition of gut microbiota in ITB was strikingly different from $\mathrm{HC}$, with the dominance of Proteobacteria and reduction of Firmicutes. Of note, the short-chain fatty acids (SCFAs)-producing bacteria such as Faecalibacterium, Roseburia, and Ruminococcus were decreased in ITB relative to $\mathrm{HC}$, while Klebsiella and Pseudomonas were enriched. Multiple predictive functional modules were altered in ITB, including the over-representation of lipopolysaccharide biosynthesis, bacterial invasion of epithelial cells, and pathogenic Escherichia coli infection that can promote inflammation. Additionally, the microbial structure in CD was distinctly different from ITB, characterized by lower alpha diversity and increased abundance of Bacteroides, Faecalibacterium, Collinsella, and Klebsiella. These four bacterial markers distinguished ITB from CD with an area under the curve of $97.6 \%$. This study established the compositional and functional perturbation of the gut microbiome in ITB and suggested the potential for using gut microbiota as biomarkers to differentiate ITB from CD.

Keywords: intestinal tuberculosis, Crohn's disease, gut microbiota, 16S rRNA sequencing, short-chain fatty acids

\section{INTRODUCTION}

Tuberculosis (TB), caused by the bacillus Mycobacterium tuberculosis, remains a public health problem and is one of the top 10 causes of death worldwide. About a quarter of the global population is infected with $M$. tuberculosis, and China is one of the 22 countries identified as having a high TB burden (World Health Organization, 2020). TB may involve any part of the body, including the lung, the primarily affected organ, and the gastrointestinal tract. Intestinal TB (ITB) is a chronic intestinal disease with non-specific clinical manifestation and endoscopic features, which were similar to Crohn's disease (CD; He Y. et al., 2019). However, the therapeutic strategies between ITB and CD are completely different. ITB patients were treated with antituberculosis medication, while $\mathrm{CD}$ patients were administered immunosuppressive agents. The misdiagnosis between 
the two diseases would either miss the best time for treatment or even worsen the condition of the patient. As the current differential diagnostic methods are complicated and timeconsuming, it is urgent to seek a convenient and efficient tool to help distinguish the two diseases in clinical practice.

With the development of high-throughput sequencing and bioinformatic analysis, there is mounting evidence that suggests the critical role of the gut microbiome in human health and disease (Feng et al., 2018). The dysbiosis of gut microbiota has been demonstrated in multiple diseases, including colorectal cancer, hepatocellular carcinoma, and inflammatory bowel disease, and the different microbial features showed the potential of disease prediction with high accuracy (Eck et al., 2017; Ren et al., 2019; Thomas et al., 2019). Several studies reported that the composition and function of gut microbiota in patients with CD were significantly different from the healthy subjects, and some of the differential microbes could classify patients by disease state with the AUC of 0.84 (He et al., 2017; Douglas et al., 2018). Our previous study also found that the mucosa-associated gut microbiota changed remarkably after the induction of remission in active $\mathrm{CD}$ patients, which indicates the potential ability of microbiota as a modality for response prediction ( $\mathrm{He} \mathrm{C}$. et al., 2019). While there have been some studies showing the gut microbiome signatures in pulmonary tuberculosis patients, the microbial profiles of ITB remain largely unclear (Luo et al., 2017; Hu et al., 2019).

To date, with the increasing incidence of $C D$ and a heavy burden of $\mathrm{TB}$ at the same time, it is of vital importance to differentiate CD and ITB, as the misdiagnosis may lead to fatal outcomes. In this study, we investigated the differences in the composition and function of the gut microbiota between ITB and healthy subjects. Furthermore, we compared the microbial communities of ITB with CD to explore the robust bacterial markers for disease discrimination. Our findings help define the gut dysbiosis of ITB and offer a novel insight into a microbiotabased model that can assist in clinical diagnosis.

\section{MATERIALS AND METHODS}

\section{Study Cohort and Sample Collection}

This study was approved by the institutional review boards of The First Affiliated Hospital of Nanchang University. Written informed consent was obtained from all the subjects involved in the study. A total of 22 patients, including 6 with ITB and 16 with active $\mathrm{CD}$, were recruited to the study cohort from the Department of Gastroenterology, The First Affiliated Hospital of Nanchang University, China. The diagnosis of ITB and $\mathrm{CD}$ was based on clinical, laboratorial, endoscopic, radiologic, and histological findings as previously described (He Y. et al., 2019). Inclusion criteria were newly diagnosed patients without the use of antibiotics, probiotics, and prebiotics for at least 1 month. Four age- and gender-matched healthy subjects without previous history of chronic disease and any drug usage were also enrolled. Seventy-one biopsy samples were collected from the ileum, ascending colon, and descending colon in each participant, although seven mucosal samples were failed for sequencing because of the small size and host contamination. All the samples were obtained during colonoscopy and frozen immediately at $-80^{\circ} \mathrm{C}$ until DNA extraction.

\section{DNA Extraction and PCR Amplification}

Total bacterial DNA was extracted from mucosal samples using the HiPure Stool DNA Kit (Magen, Guangzhou, China) according to the instructions of the manufacturer. The quality and concentration of DNA were detected using NanoDrop 2000C spectrophotometer (Thermo Fisher Scientific, Waltham, MA, United States). The DNA integrity was evaluated by $1 \%$ agarose gel electrophoresis. The V3-V4 regions of the $16 \mathrm{~S}$ rRNA gene were amplified by PCR using primers 341F 5'-CCTACGGGNGGCWGCAG-3' and 806R 5'GGACTACHVGGGTATCTAAT-3'. Genomic DNA was initially denatured at $95^{\circ} \mathrm{C}$ for $2 \mathrm{~min}$, followed by 27 cycles consisting of denaturation at $98^{\circ} \mathrm{C}$ for $10 \mathrm{~s}$, annealing at $62^{\circ} \mathrm{C}$ for $30 \mathrm{~s}$, elongation at $68^{\circ} \mathrm{C}$ for $30 \mathrm{~s}$, and a final extension step at $68^{\circ} \mathrm{C}$ for $10 \mathrm{~min}$. PCRs were performed in triplicate, $50 \mu \mathrm{l}$ of a mixture containing $5 \mu \mathrm{l}$ of $10 \times$ KOD buffer, $5 \mu \mathrm{l}$ of $2.5 \mathrm{mM}$ dNTPs, $1.5 \mu \mathrm{l}$ of $5 \mu \mathrm{M}$ primer, $1 \mu \mathrm{l}$ of KOD polymerase, and $100 \mathrm{ng}$ of template DNA.

\section{S rRNA Gene Sequencing}

The PCR amplicons were extracted from 2\% agarose gels and purified using the AxyPrep DNA Gel Extraction Kit (Axygen Biosciences, Union City, CA, United States) according to the protocols of the manufacturer, and they were quantified using the QuantiFluor-ST (Promega, Madison, WI, United States). Purified amplicons were pooled in equimolar quantities, and a sequencing library was constructed according to the official details of the Illumina. Subsequently, next-generation sequencing was performed using the Illumina Hiseq 2500 platform (Illumina, San Diego, CA, United States) by Genedenovo Biotechnology Co., Ltd. (Guangzhou, China) with $2 \times 250$ bp paired-end reads.

\section{Bioinformatics Analysis}

Raw reads were filtered using FASTP (version 0.18.0): (1) remove reads containing more than $10 \%$ of unknown nucleotides and (2) remove reads containing less than $50 \%$ of bases with quality (Q-value) > 20 (Chen et al., 2018). Paired and clean reads were merged as raw tags using FLASH (version 1.2.11) with a minimum overlap of $10 \mathrm{bp}$ and a mismatch error rate of 2\% (Magoc and Salzberg, 2011). High-quality clean tags were obtained by QIIME (version 1.9.1) pipeline, and then, the chimera checking was performed based on the reference ${ }^{1}$ using UCHIME algorithm. ${ }^{2}$ The effective tags were clustered into operational taxonomic units (OTUs) with similarity $\geq 97 \%$ using UPARSE pipeline (Edgar, 2013). The tag sequence with the highest abundance was selected as a representative sequence. The representative OTUs were classified into organisms by a naive Bayesian model using the RDP classifier (version 2.2) based on the SILVA database (version 132) with the confidence threshold value of 0.8 (Wang et al., 2007).

\footnotetext{
${ }^{1}$ http://drive5.com/uchime/uchime_download.html

${ }^{2} \mathrm{http}: / /$ www.drive5.com/usearch/manual/uchime_algo.html
} 
The abundance statistics of each taxonomy was visualized using Krona (version 2.6) (Ondov et al., 2011). The stacked bar plot of the community composition was visualized in $\mathrm{R}$ project ggplot2 package (version 2.2.1). The alpha diversity indexes including Sobs, Shannon, and Chaol were calculated in QIIME (Thukral, 2017). The beta diversity was estimated by the weighted UniFrac and Bray-Curtis distances and visualized with principal coordinates analysis (PCoA) in R project Vegan package (version 2.5.3). Species comparison between groups was performed by Welch's $t$-test, and biomarker features were screened by randomForest package (version 4.5 .12 ) in $\mathrm{R}$. The KEGG pathway analysis of the OTUs was inferred using PICRUSt (version 2.1.4) to illustrate the predictive functional profiling of microbial communities (Langille et al., 2013).

\section{Statistical Analysis}

Statistical analyses of demographic characteristics were performed using a one-way ANOVA for age and body mass index (BMI) and using chi-square test with Statistical Product and Service Solutions (SPSS) 20.0 (IBM) for gender. The comparison of alpha diversity indexes was calculated by Welch's $t$-test. Statistical analysis of beta diversity between different groups was conducted by Adonis test. $P$-value $<0.05$ was considered as statistically significant. Bioinformatic analysis was performed using Omicsmart, a dynamic real-time interactive online platform for data analysis. ${ }^{3}$

\section{RESULTS}

Demographic characteristics of the subjects included in this study were shown in Supplementary Table 1. There was no significant difference of age, gender, and BMI among patients with $\mathrm{CD}$, ITB, and healthy controls (HC). In order to get a comprehensive profile of the gut microbiota, mucosal samples were collected from three intestinal segments of each subject, including ileum, ascending colon, and descending colon, and then, 16S rRNA gene sequencing was performed. A total of

\footnotetext{
${ }^{3}$ http://www.omicsmart.com
}

A

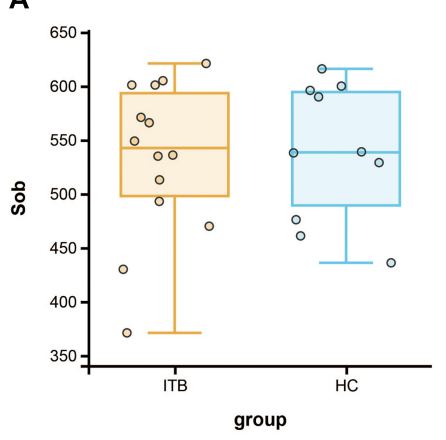

D

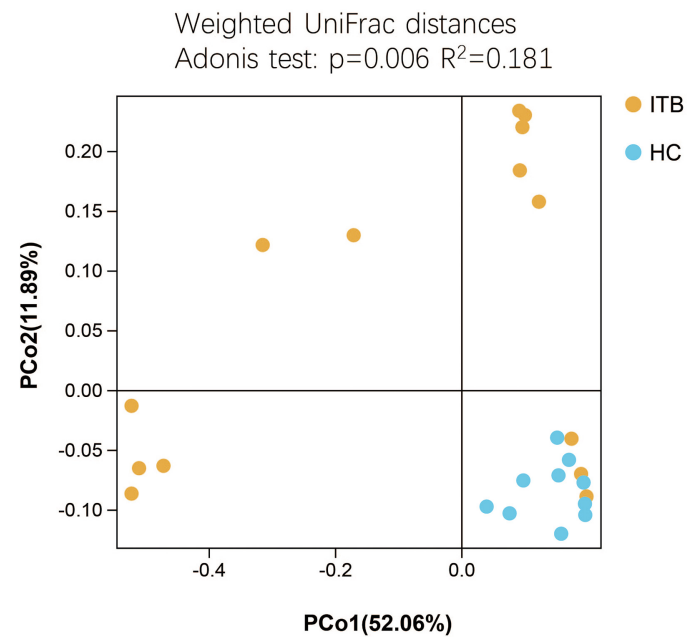

B

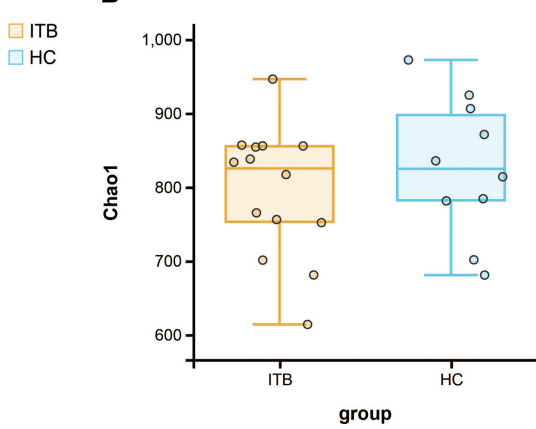

C

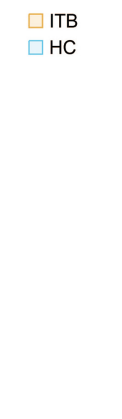

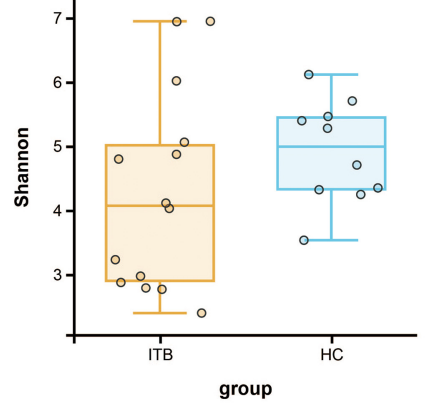




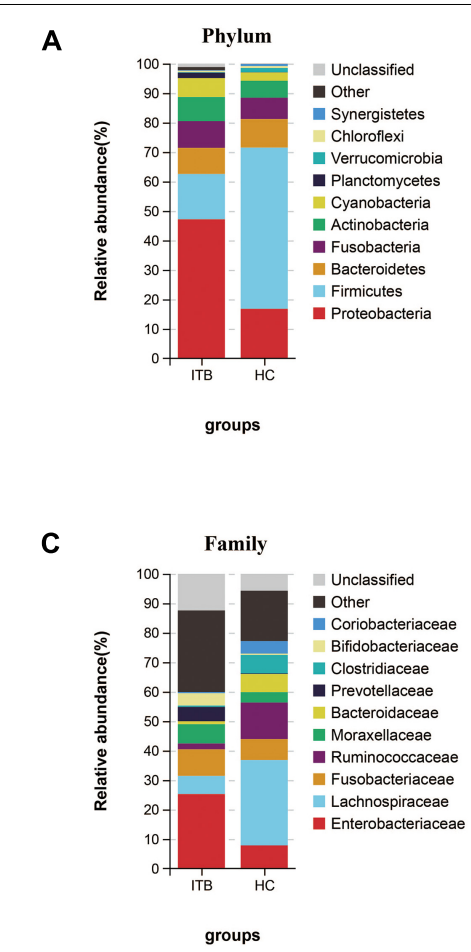

E

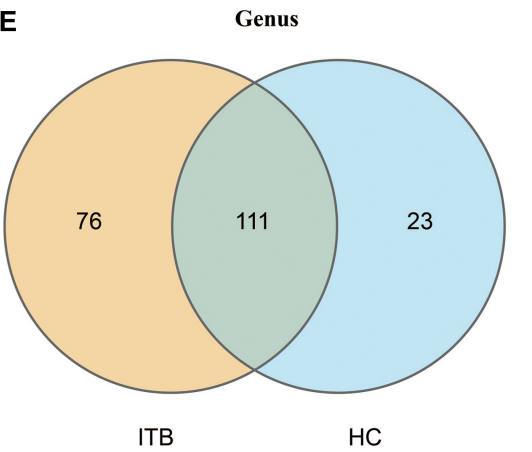

B

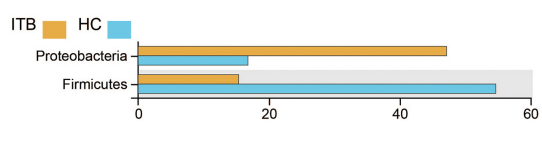

Mean abundance(\%)

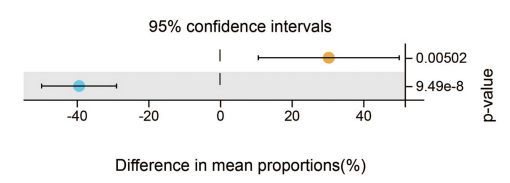

D ITB $\mathrm{HC}$
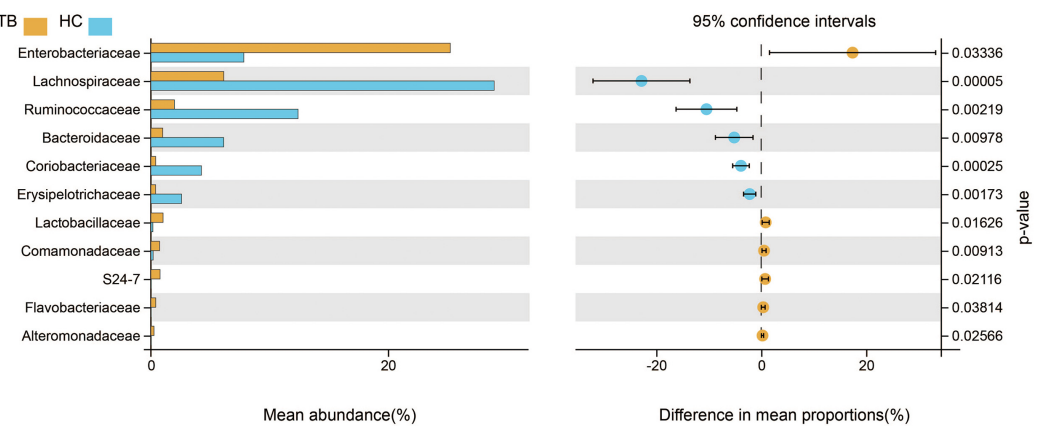

F

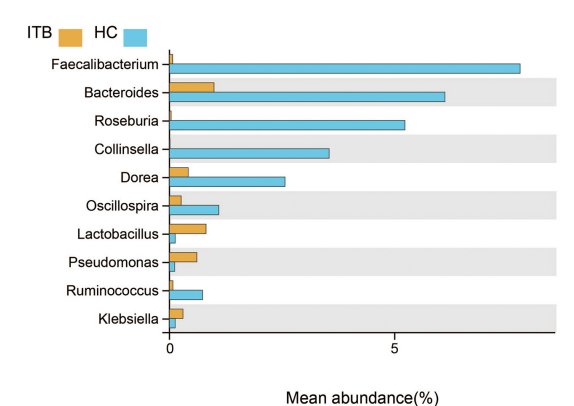

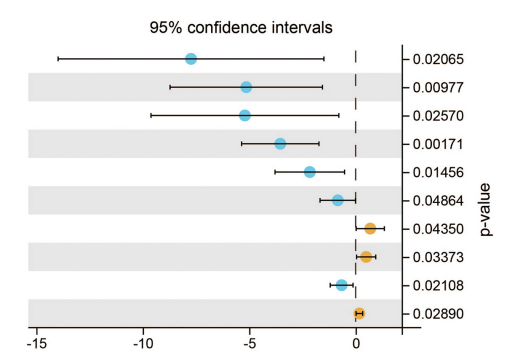

Difference in mean proportions(\%)

FIGURE 2 | Variations of gut microbiota composition in ITB versus HC. Relative proportions of the top 10 most abundant bacteria at the phylum (A) and family (C) level in ITB and HC. The differentially abundant taxa between ITB and HC are identified at the phylum (B), family (D), and genus (F) level using Welch's $t$-test.

(E) Venn diagram showed the shared and unique bacterial genera between these two groups. ITB, intestinal tuberculosis; HC, healthy controls.

$8,769,199$ raw sequences were obtained. After quality filtering and binning, 8,048,679 sequences were retained for further analysis with an average of 113,361 sequences per sample (range 28,794224,414 sequences/sample).

\section{The Microbial Features in ITB Compared With HC}

Microbial alpha diversity analysis revealed that the indexes including observed species (Sob), Chaol, and Shannon were not significantly different between ITB and HC (Figures 1A-C). We next assessed the dissimilarities between ITB and HC using the Bray-Curtis dissimilarity and weighted UniFrac distance metrics to evaluate the overall differences in beta diversity. The PCoA showed that samples of ITB clustered separately from those of HC (Figures 1D,E). The $p$-values obtained using the Adonis test for both distances were significant $(p<0.01)$, and the $R^{2}$ values were indicative of remarkable variance between ITB and HC.

To investigate the specific changes of microbiota in samples of ITB, we assessed the relative abundance of taxa in ITB and HC. As shown in Figure 2A, the Firmicutes, Bacteroidetes, and Proteobacteria were the top three most abundant phyla, with differential distribution between ITB and HC. While Firmicutes were highly abundant in HC (54.7\%), patients with ITB were dominated by Proteobacteria (47.2\%) with a lower abundance of Firmicutes (15.4\%) (Figure 2B). At the family level, the most abundant taxa included Enterobacteriaceae, Lachnospiraceae, Fusobacteriaceae, and Ruminococcaceae. Compared with HC, 


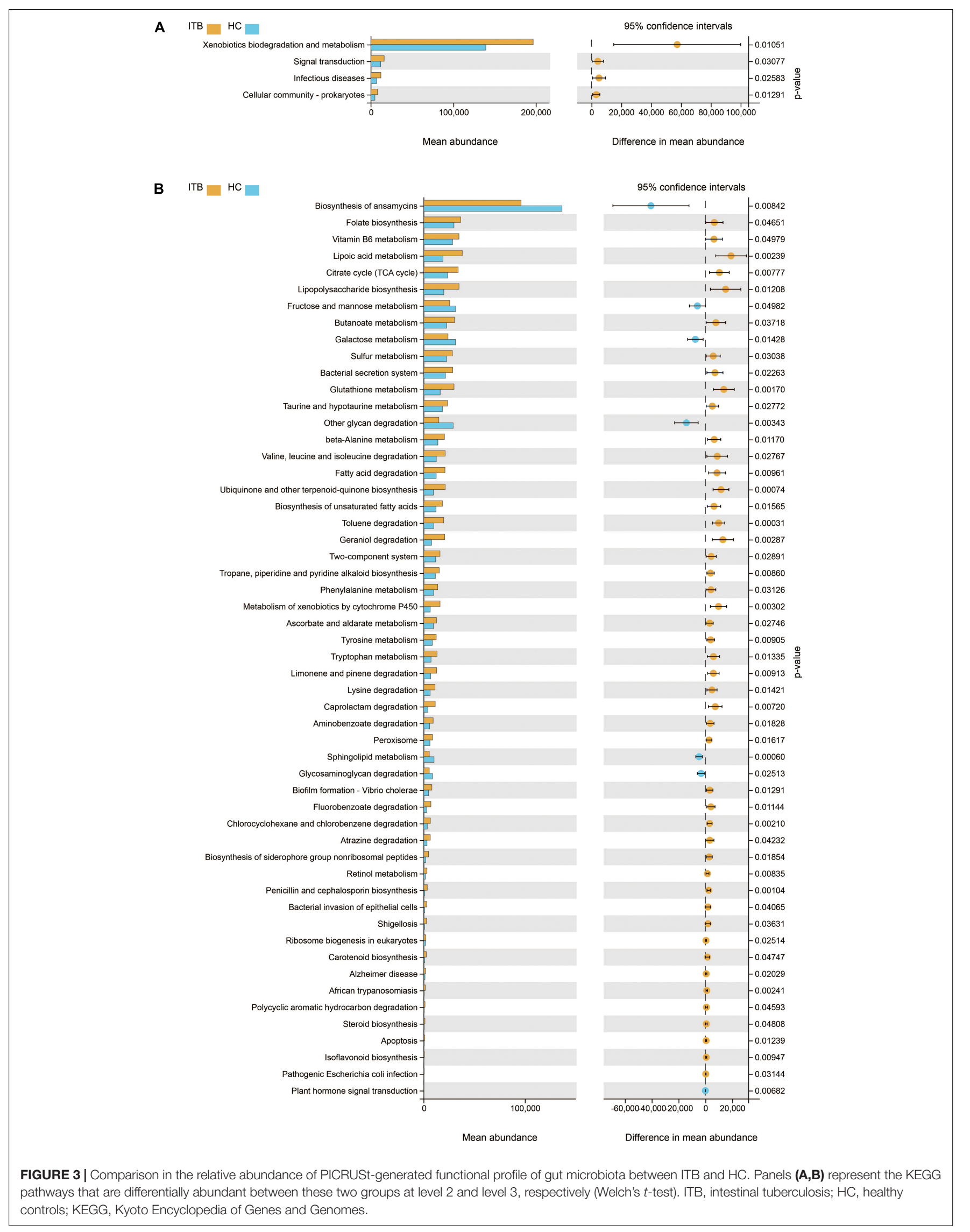


the relative abundance of Enterobacteriaceae belonging to Proteobacteria was higher in ITB, whereas Lachnospiraceae and Ruminococcaceae were prominently decreased which may lead to the reduction of Firmicutes (Figures 2C,D). There were 76 and 23 genera that were uniquely found in ITB and $\mathrm{HC}$, respectively, while 111 genera were in common (Figure 2E and Supplementary Table 2). Of the 111 genera, we observed 10 bacterial taxa that displayed different abundance between ITB and HC (Figure 2F). Three genera including Lactobacillus, Pseudomonas, and Klebsiella were overrepresented in ITB compared with HC. Conversely, seven genera (Faecalibacterium, Bacteroides, Roseburia, Collinsella, Dorea, Oscillospira, and Ruminococcus) were significantly decreased in ITB. Although M. tuberculosis was not detected in ITB at the species level, the genus Mycobacterium was more abundant in ITB compared with HC (Metastats analysis, $p=0.014$ ). To assess the diagnostic value of gut microbial markers for ITB, we used genus information to construct a random forest classifier model between ITB and HC. Two specific importance measures including the mean decrease accuracy and the mean decrease in Gini were performed, and the top three most important genera in common (Faecalibacterium, Roseburia, and Collinsella) were chosen for subsequent analysis (Supplementary Figures 1A,B). The testing result showed that these three features were able to differentiate patients with ITB from HC with high accuracy (AUC = 1, 95\% CI 1-1, Supplementary Figure 1C).

Furthermore, we used PICRUSt to infer the metagenome functional content based on the microbial community profiles obtained from the 16S rRNA gene sequences. The functional changes in ITB microbiomes included significantly increased representation of predicted KEGG pathways of level 2 involved in xenobiotics biodegradation and metabolism, signal transduction, infectious diseases (Figure 3A). The level 3 KEGG pathway data indicated that lipopolysaccharide biosynthesis, bacterial secretion system, bacterial invasion of epithelial cells, shigellosis, and pathogenic Escherichia coli infection were enriched in ITB compared with HC. In contrast, the gut microbiome of HC was characterized by over-representation of physiological pathways, including fructose and mannose metabolism, galactose

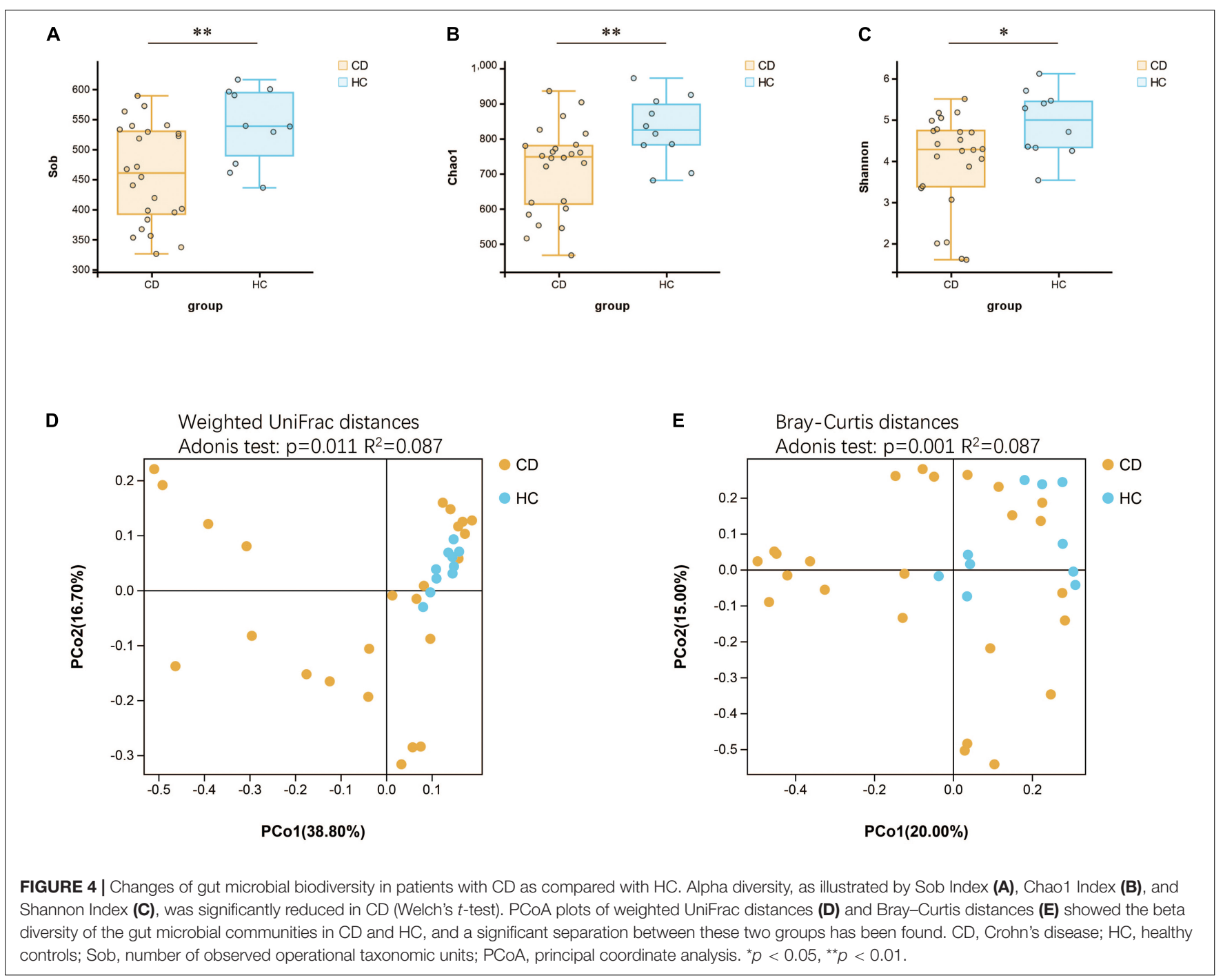




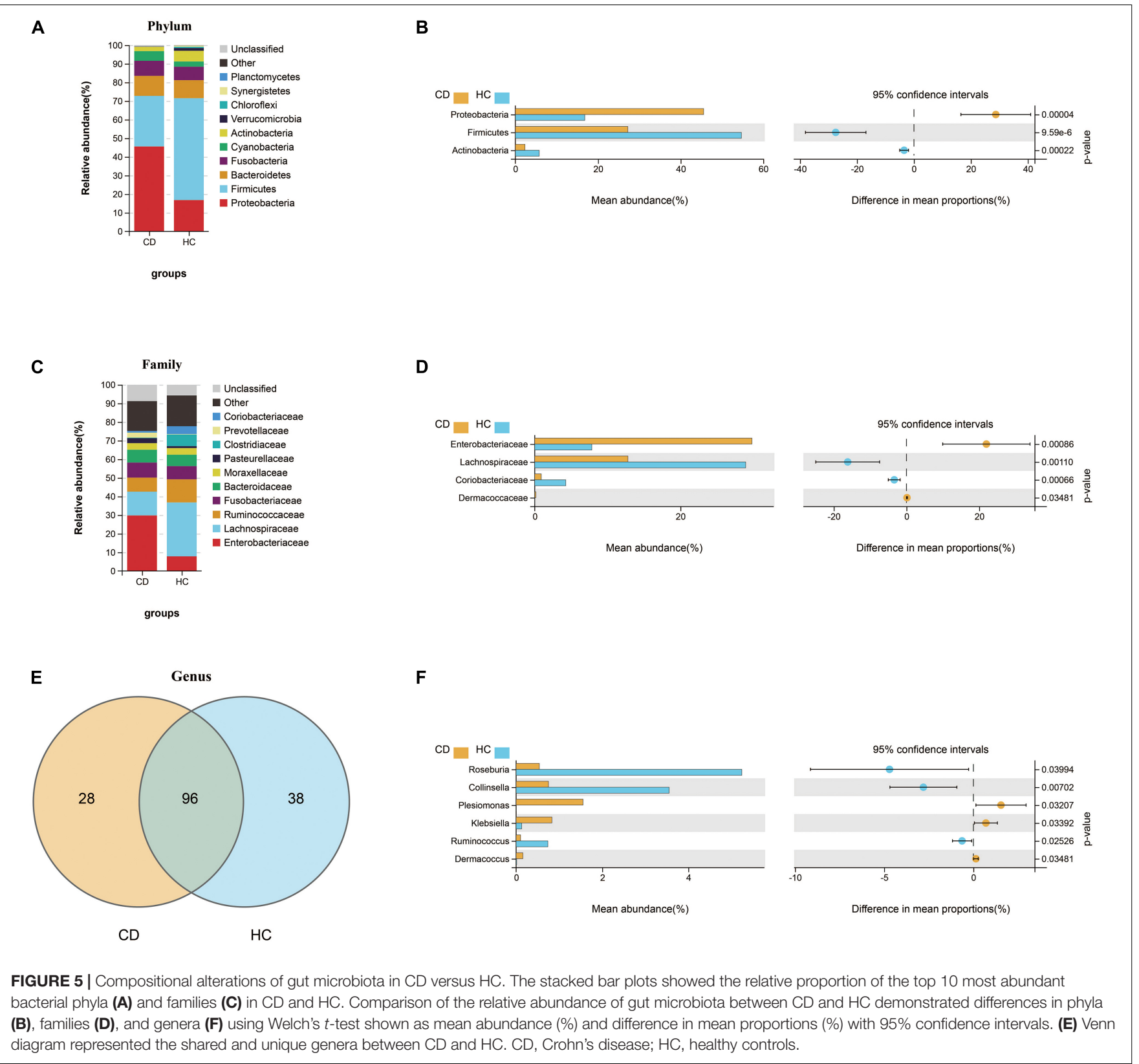

metabolism, other glycan degradation, sphingolipid metabolism, and glycosaminoglycan degradation (Figure 3B).

\section{The Alterations of Gut Microbiota in Active CD in Comparison With HC}

Initially, the gut microbiota richness, as measured by Sob, Shannon, and Chaol indexes, was reduced in patients with active $\mathrm{CD}$ as compared with $\mathrm{HC}$ (Figures $\mathbf{4 A - C}$ ). Then, we sought to explore whether the overall bacterial phenotypes of $\mathrm{CD}$ and $\mathrm{HC}$ were different. Beta diversity was calculated using both the weighted UniFrac distances and Bray-Curtis distances and visualized in PCoA plots. The total diversity captured by the top two principal coordinates was 55.5 and $35 \%$ for the weighted
UniFrac and Bray-Curtis distances, respectively. The microbiota composition of active $\mathrm{CD}$ was distinctly different from that of $\mathrm{HC}$ (Adonis test, $p=0.011, R^{2}=0.087$ for weighted distances, and $p=0.001, R^{2}=0.087$ for Bray-Curtis distances, Figures $\left.4 \mathrm{D}, \mathrm{E}\right)$.

Next, we investigated the compositional differences between active $\mathrm{CD}$ and $\mathrm{HC}$ at three microbial levels. Interestingly, patients with $\mathrm{CD}$ harbored increased abundance of Proteobacteria (45.6\%) and decreased abundance of Firmicutes (27.2\%), the changes of which were quite similar to ITB as compared with HC (Figures 5A,B). At the family level, the relative abundance of Enterobacteriaceae was upregulated in $\mathrm{CD}$ compared with HC, while Lachnospiraceae and Coriobacteriaceae were downregulated (Figures 5C,D). A total of 162 genera were identified and 96 genera were shared in both $\mathrm{CD}$ and $\mathrm{HC}$ 
A $\mathrm{CD} \quad \mathrm{HC}$

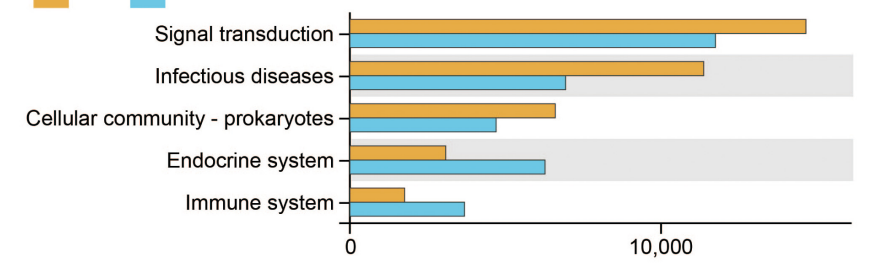

Mean abundance

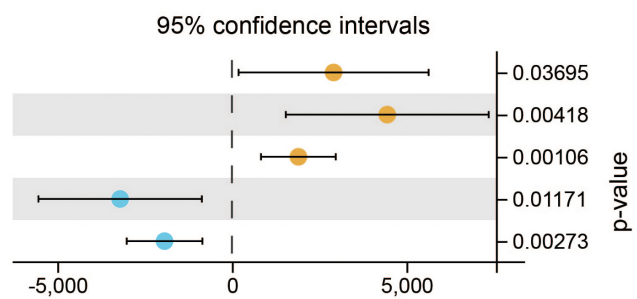

Difference in mean abundance
B $\mathrm{CD} \quad \mathrm{HC}$

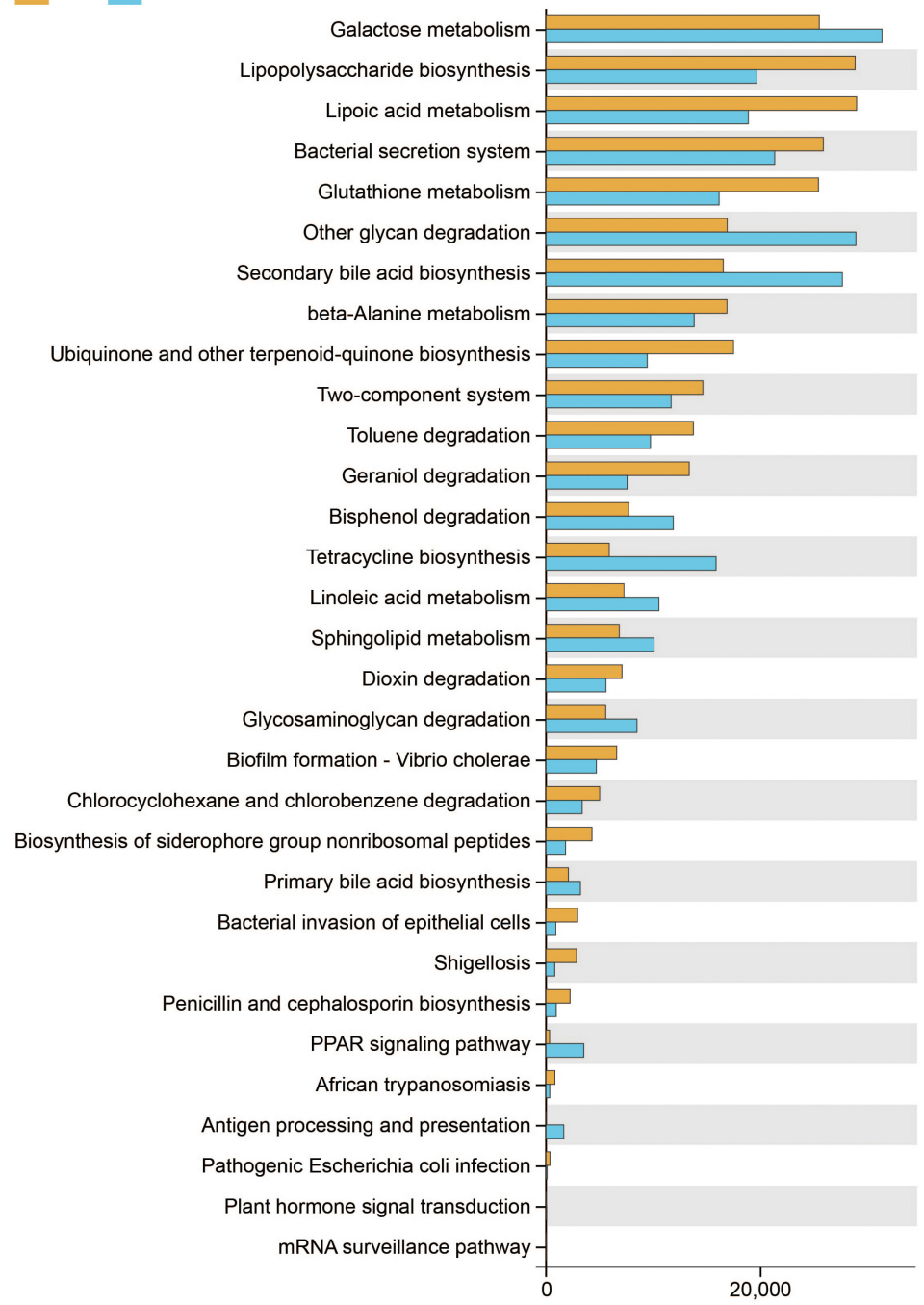

Mean abundance

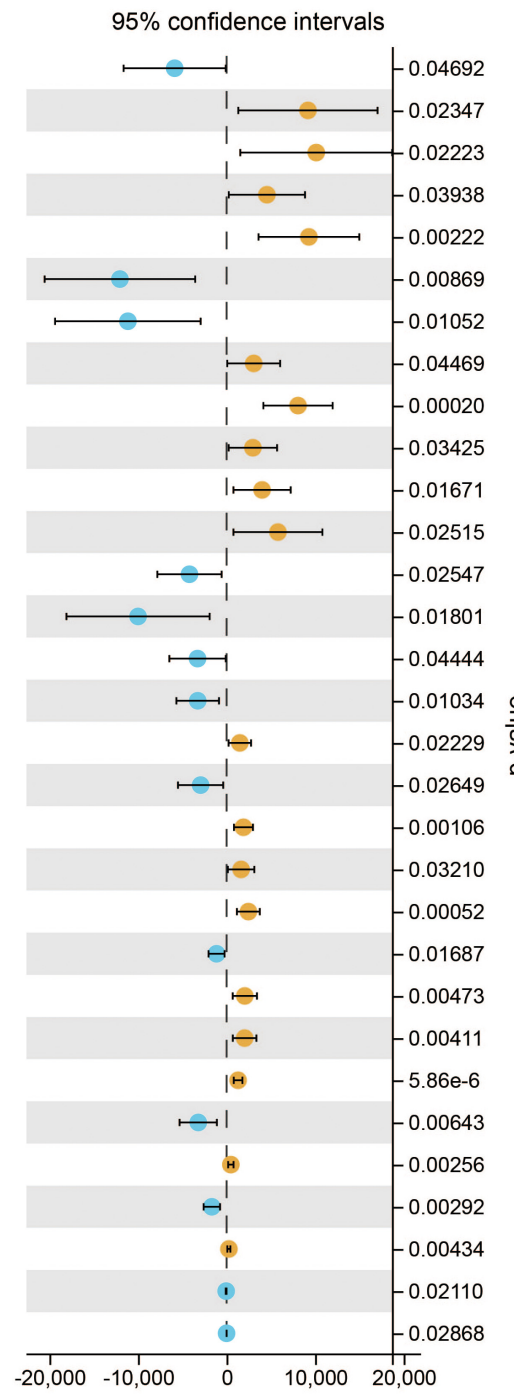

Difference in mean abundance

FIGURE 6 | Functional features of gut microbiota in CD. The functional composition of gut microbiota based on PICRUSt prediction was compared between CD and HC using Welch's t-test. (A) KEGG pathway at level 2 and (B) KEGG pathway at level 3. CD, Crohn's disease; HC, healthy controls; KEGG, Kyoto Encyclopedia of Genes and Genomes. 


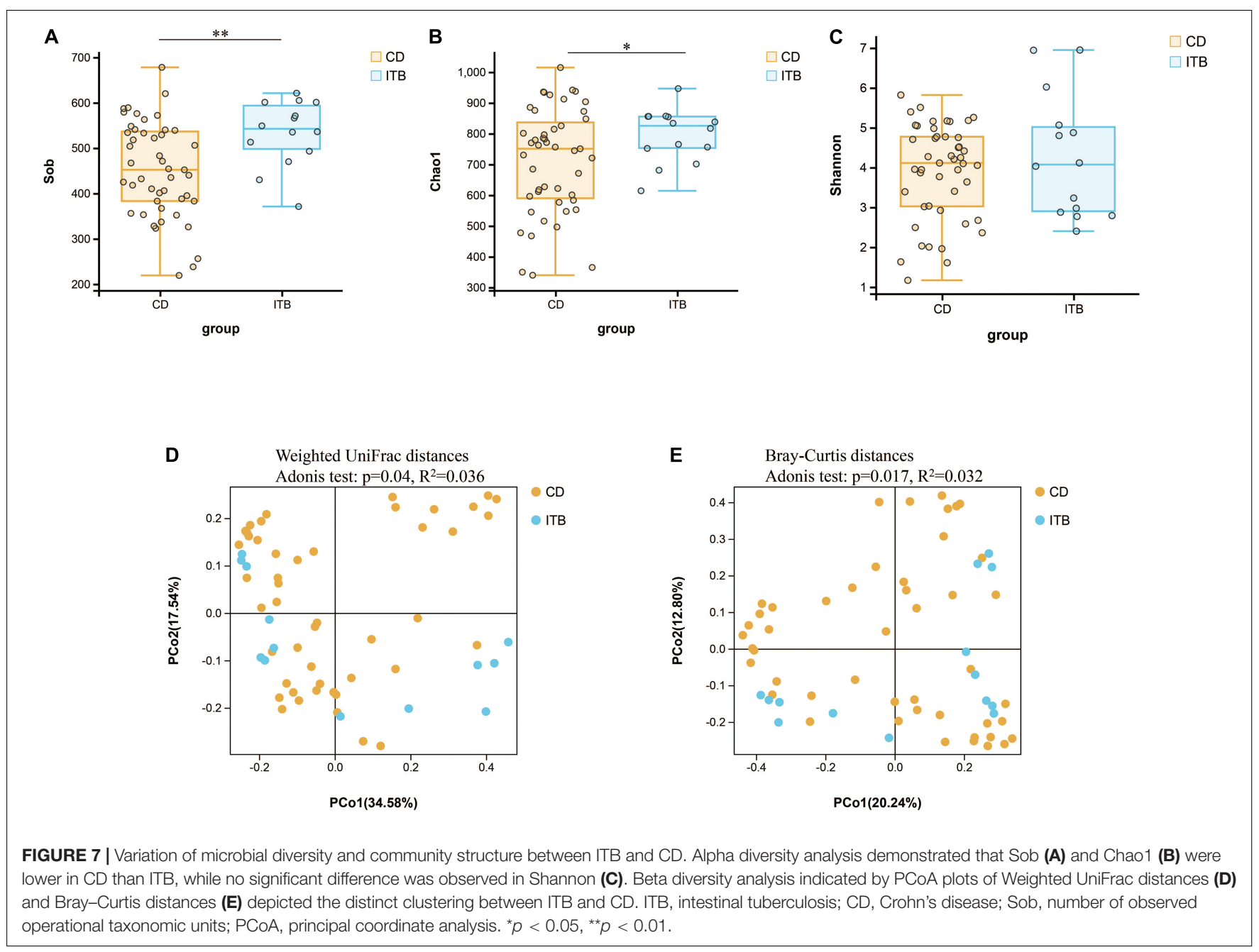

(Figure 5E). Of these, 28 genera were unique to $\mathrm{CD}$ and 38 genera were unique to HC (Supplementary Table 3). Relative to $\mathrm{HC}$, patients with active CD displayed a higher abundance of Klebsiella, Plesiomonas, and Dermacoccus and a lower abundance of Roseburia, Collinsella, and Ruminococcus (Figure 5F). To explore the diagnostic value of gut microbiota for $\mathrm{CD}$, we constructed a random forest classifier model to distinguish CD patients from HC. Three genera including Collinsella, Roseburia, and Haemophilus were finally selected based on both the mean decrease Gini and the mean decrease accuracy measures (Supplementary Figures 2A,B). We applied the receiver operating characteristic (ROC) curve and found that the 3-genera set could separate CD from HC with an AUC of 87.5\% (95\% CI 0.752-0.998) (Supplementary Figure 2C).

We further studied the functional changes of microbial communities between $\mathrm{CD}$ and $\mathrm{HC}$ using PICRUSt. At KEGG level 2, we found that signal transduction and infectious diseases were enriched in CD compared with $\mathrm{HC}$, while the endocrine system and immune system were inactivated (Figure 6A). Of note, functional pathways at level 3 including lipopolysaccharide biosynthesis, bacterial secretion system, bacterial invasion of epithelial cells, shigellosis, and pathogenic E. coli infection were much abundant in CD. In contrast, the physiological modules such as galactose metabolism, other glycan degradation, secondary bile acid biosynthesis, and linoleic acid metabolism were depleted in CD (Figure 6B).

\section{The Distinct Microbial Characteristics to Differentiate Between ITB and CD}

Despite the growing body of studies depicting the gut microbiome profiling in patients with $\mathrm{CD}$, there is a paucity of literature exploring whether the structure of gut microbiota is different between CD and ITB. First, the analysis of alpha diversity revealed that both the richness and diversity as calculated in Sob and Chaol were lower in CD compared with ITB, while no significant difference was observed in Shannon Index (Figures 7A-C). The analysis of beta diversity as calculated on the Bray-Curtis dissimilarity and weighted UniFrac distances showed the mucosal-associated microbial community of ITB samples apart from that of CD (Figures 7D,E), although the overall compositional difference between ITB 


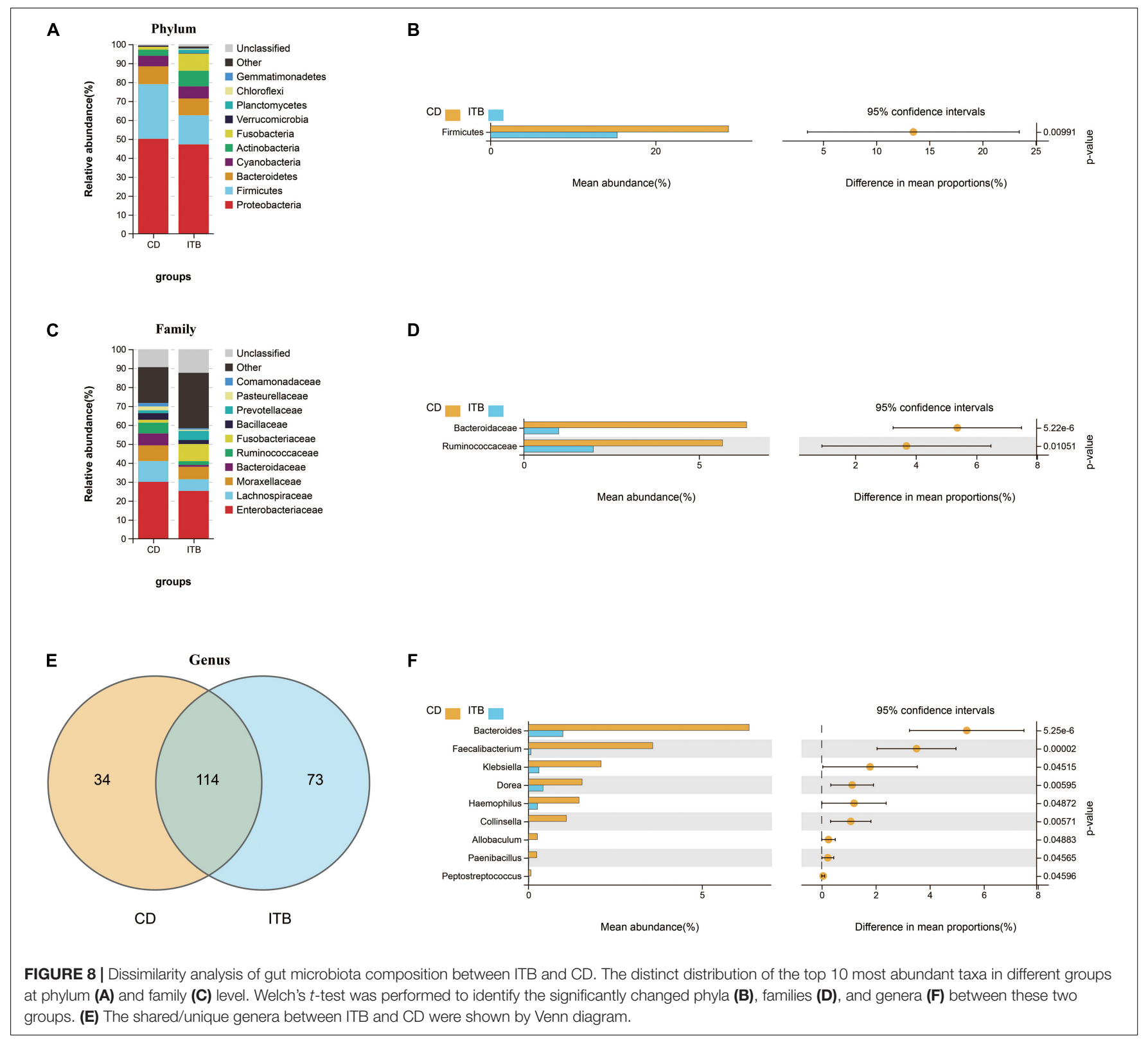

and $\mathrm{CD}$ was not as striking as the distinction between either disease and $\mathrm{HC}$.

Intergroup comparisons of taxonomic profiles revealed that samples from ITB and CD exhibited alterations in the abundance of several taxa. At the phylum level, the relative abundance of Firmicutes was lower in ITB compared with CD (Figures 8A,B). With regard to the family level, Ruminococcaceae belonged to Firmicutes as well as Bacteroidaceae were significantly reduced in ITB compared with CD (Figures 8C,D). While 114 genera were shared by both ITB and CD, there were 34 genera and 73 genera that were exclusively found in CD and ITB, respectively (Figure 8E and Supplementary Table 4). Further statistical analysis showed a decreased abundance of genera, including Bacteroides, Faecalibacterium, and Klebsiella, in ITB compared with CD (Figure 8F). To explore the potential ability of the gut microbiome to discriminate ITB from $\mathrm{CD}$, we used the machine learning method Random Forest to the genus level dataset. Figure 9A showed the top 20 features ordered by two specific importance measures: the mean decrease accuracy and the mean decrease in Gini. Of these, four genera (Collinsella, Bacteroides, Faecalibacterium, and Klebsiella) were chosen based on both Gini Index and relative abundance. The performance of the model was assessed using ROC analysis, and the testing results showed that Collinsella, Bacteroides, Faecalibacterium, and Klebsiella individually achieved an accuracy of 0.83 (95\% CI, 0.72-0.94), 0.79 (95\% CI, 0.67-0.91), 0.77 (95\% CI, 0.650.89 ), and 0.57 (95\% CI, 0.43-0.72), respectively (Figure 9B). Of interest, the combination of these four candidate biomarkers improved the accuracy of differentiation between ITB and CD to 0.976 (95\% CI, 0.93-1) (Figure 9C). 
A

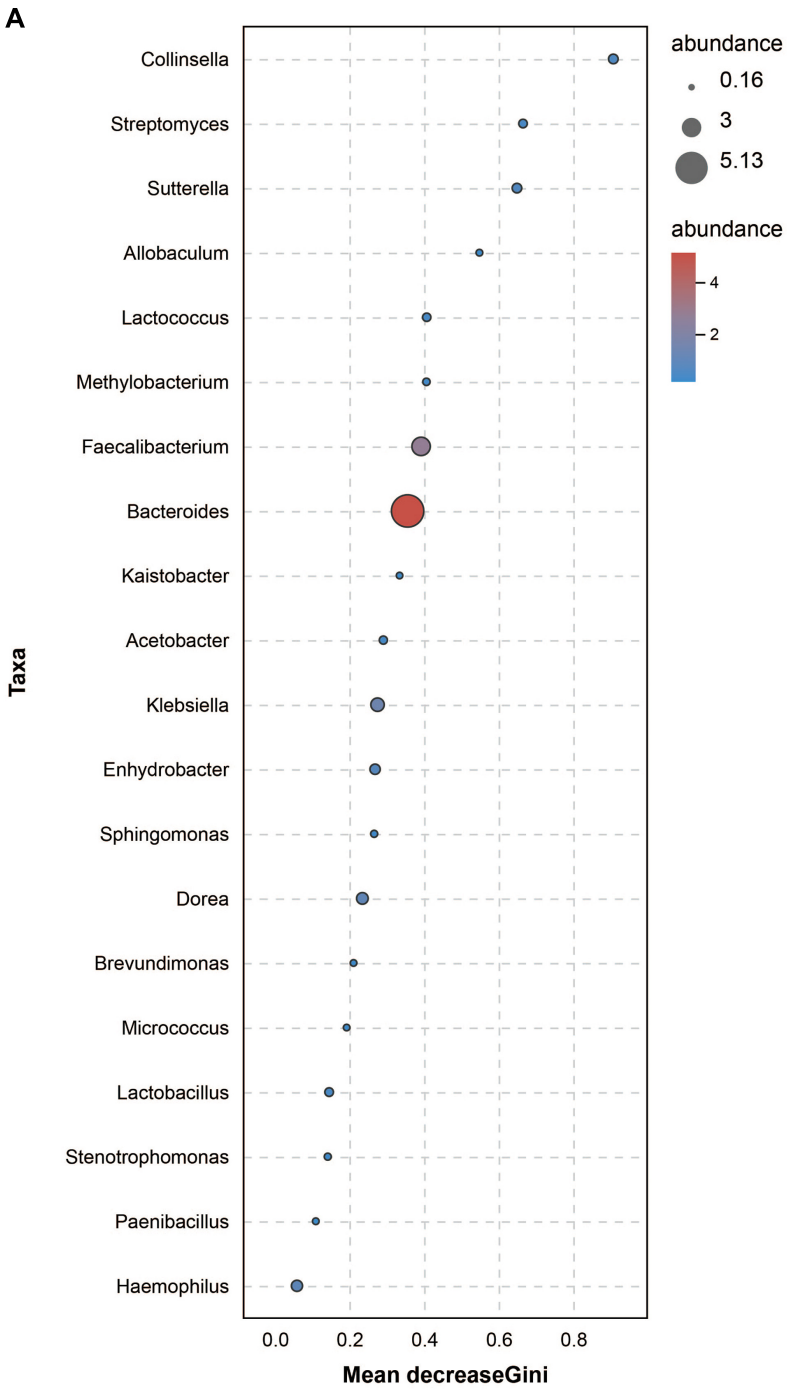

B
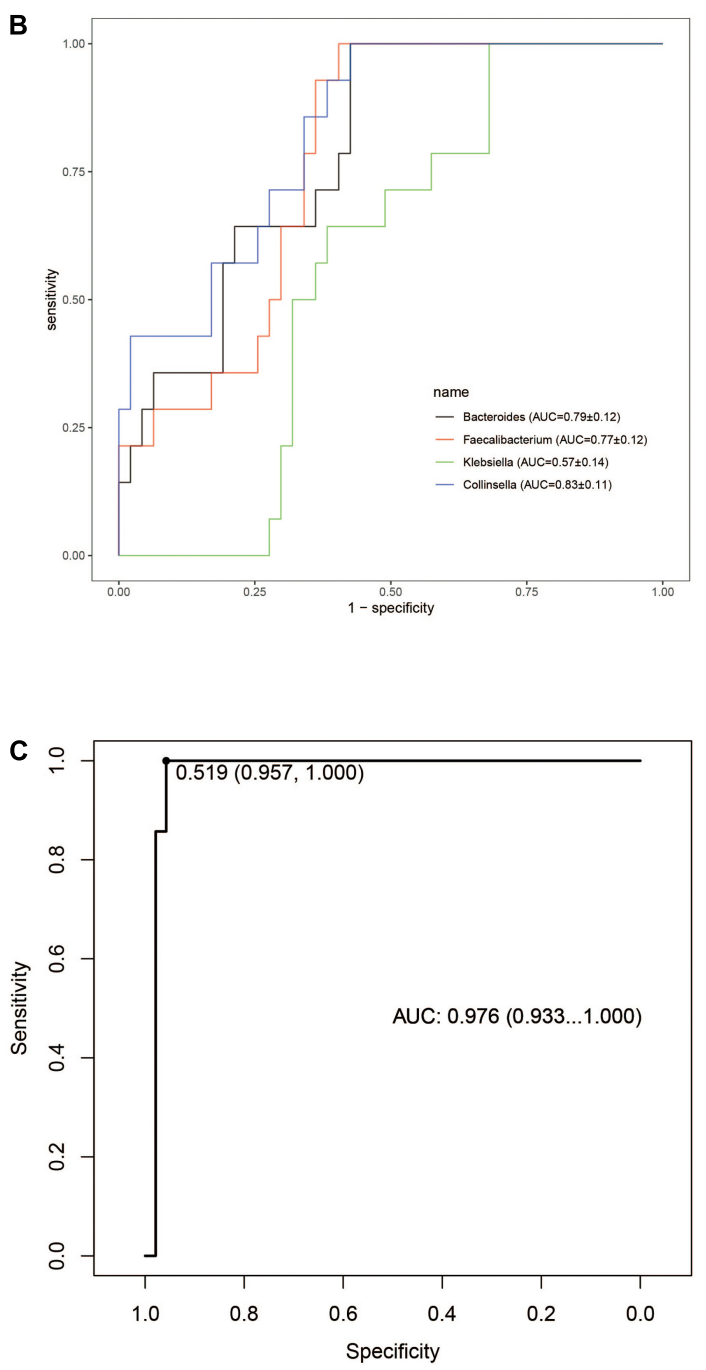

FIGURE 9 | Gut microbiota biomarkers can be used to discriminate ITB from CD using random forest models. (A) The top 20 genera were detected for the classification between ITB and CD. Four biomarker taxa were identified based on mean decrease Gini Index and relative abundance. (B) The individual bacterial candidate could discriminate the two groups with AUC ranging from 0.57 to 0.83 . (C) The combination of these four genera yielded more robust diagnostic performance over that of separate genera.

\section{DISCUSSION}

Since ITB and CD share similar clinical, endoscopic, and histological features, the differentiation between these two diseases is a challenge for clinicians, especially in developing countries, where the prevalence of ITB remains high and the incidence of CD is rising (Kedia et al., 2019). Although several predictive models have been developed and validated with good accuracy, most of them involved multiple parameters, and thus, it is complicated and time-consuming for clinical application (Limsrivilai and Pausawasdi, 2021). With the development of high-throughput sequencing and bioinformatic analysis, accumulating evidence has demonstrated the great capability of the gut microbiota as a diagnostic tool for human diseases (Lopetuso et al., 2018). Herein, we delineated the community structure of mucosa-associated microbiome in patients with newly diagnosed ITB by means of $16 \mathrm{~S}$ rRNA gene sequencing. Our data demonstrated that ITB was associated with altered composition and function of gut microbiota, including the decreased abundance of Faecalibacterium and Roseburia and the increased abundance of Klebsiella and Lactobacillus. Moreover, we found that the microbial phenotype of ITB was distinctly different from active $\mathrm{CD}$, although these two intestinal diseases shared some bacterial changes in common as compared with HC. Based on the microbial signature, we established a model containing four genera that have discriminatory power for differentiating ITB from CD.

So far, numerous studies have investigated the relationship between pulmonary infection of $M$. tuberculosis and gut microbiome, yet the alterations of gut microbiota in ITB are 
obscure. In this study, we found that patients with ITB host a markedly different mucosa-associated gut microbiome, with a significant shift in the global diversity. The intestinal dysbiosis of ITB was defined by the dominance of Proteobacteria, which contain many pathogenic species, as well as the depletion of Firmicutes. Further differential analysis at the genus level showed that the relative abundance of Faecalibacterium, Roseburia, and Ruminococcus, which are recognized as the short-chain fatty acids (SCFAs)-producing microbes, was dramatically decreased in ITB. Interestingly, the reduction of SCFAs-producing bacteria was also observed in the stool samples of patients with pulmonary tuberculosis, indicating the vital role of SCFAs in the infection of M. tuberculosis (Luo et al., 2017; Hu et al., 2019). SCFAs, as a major group of metabolites from gut microbes, are known to exert a beneficial effect on health through regulating innate immunity and protecting gut barrier integrity (Liu et al., 2021). We speculated that the reduction of genus Faecalibacterium and Roseburia in ITB might lead to the impaired production of SCFAs and the consequent intestinal metabolic disorders. Additionally, we observed the over-growth of pathogenic Klebsiella and Pseudomonas in ITB, which may contribute to the activation of several bacterial-associated pathways as revealed by functional prediction, such as bacterial invasion of epithelial cells, lipopolysaccharide biosynthesis, and pathogenic E. coli infection. Taken together, the loss of beneficial microbes as well as the enrichment of opportunistic pathogen could finally promote the intestinal injury in ITB.

It is widely established that the gut microbiota plays an important role in the development of CD in both human and animal models (Pascal et al., 2017; Wu et al., 2020). In our research, the mucosa-associated microbiome of patients with active $\mathrm{CD}$ exhibited decreased species richness and evenness compared with $\mathrm{HC}$, which agrees with previous studies (Wang et al., 2017). Low microbial diversity, which has been reported in a variety of human diseases, is considered as one of the major types of gut dysbiosis (Kriss et al., 2018). Moreover, our previous study demonstrated that the bacterial diversity in patients with active $C D$ was significantly increased after the remission induction therapy, which suggests the association between microbial diversity and disease activity ( $\mathrm{He} \mathrm{C}$. et al., 2019). Recently, several studies have reported that CD patients displayed lower relative abundances of SCFA-producing bacteria, which were correlated with the reduction of SCFAs in fecal samples (Wang et al., 2017; Wang Y. et al., 2021). In line with this finding, we also observed the decreased abundance of several SCFAs producers including Roseburia and Ruminococcus in CD relative to HC. Additionally, Collinsella has been identified as one of the microorganisms that could be used to discriminate $\mathrm{CD}$ from non-CD patients, with lower relative abundance in $\mathrm{CD}$ (Pascal et al., 2017). This agrees with the observation in this study.

One of the novel findings of this study was that the structure of gut microbiota in ITB and CD patients showed both common and different characteristics. Of interest, the predominant bacteria at the phylum level were Proteobacteria in both ITB and CD instead of Firmicutes in HC. Furthermore, we found that the reduction of Firmicutes in ITB and CD was attributed to the depletion of genus Roseburia and Ruminococcus, which were known as
SCFAs producers. In addition to the bacterial composition, the functional analysis also showed that the pathways including the bacterial secretion system, bacteria invasion of epithelial cells, and pathogenic E. coli infection were significantly enriched in both ITB and CD relative to $\mathrm{HC}$. We speculated that similar alterations of gut microbiota in ITB and CD might contribute to the resemblance of the endoscopic manifestations of the intestinal injury. However, there are some distinctive characteristics of gut microbiota in ITB and CD. Patients with CD exhibited lower microbial diversity relative to ITB. While the SCFAs producers including family Ruminococcaceae and genus Faecalibacterium were reduced in both ITB and CD as compared with HC, their abundance was much lower in ITB than CD. Moreover, we found that the widely reported SCFAsproducing bacteria such as Faecalibacterium, Clostridium, and Bifidobacterium were significantly increased after 6 months of antituberculosis treatment (Supplementary Figure 3). Thus, the abundance of SCFAs-producing bacteria as well as their metabolites may play a critical role in modulating immune and inflammatory response against tuberculosis, which is in concordant with previous reports (Wang S. et al., 2021).

Several limitations of this study should be noted. Although we first explored the alterations of gut microbiota in patients with ITB as well as the microbial biomarkers to differentiate ITB from CD, the number of patients was relatively small, and the predictive efficacy of bacterial candidate warrants validation in large-scale multicenter studies. Additionally, the imbalance of gut microbiota assessed by $16 \mathrm{~S}$ rRNA gene sequencing in this study needs to be confirmed through shotgun microbiome metagenomics, which may reveal more accurate taxonomic composition and function. Finally, we investigated the changes of mucosal-associated microbiota in ITB and CD, which were probably different from fecal microbiota. As fecal samples are more readily obtained, the use of fecal bacteria as markers to distinguish ITB from CD will be determined in our future study.

\section{CONCLUSION}

Our data provide a detailed description of the disruption of mucosa-associated microbiota in patients with ITB, which was characterized by the dominance of Proteobacteria and a dramatic loss of SCFA-producing bacteria such as Faecalibacterium, Roseburia, and Ruminococcus. We also observed the distinction and similarity of microbial features between ITB and CD, two intestinal diseases that are difficult to distinguish for clinicians. Biomarkers based on four mucosal bacteria can discriminate ITB from CD with good performance. Additional studies are warranted to validate the potential capability of gut microbiota as a convenient tool for the differentiation between ITB and CD.

\section{DATA AVAILABILITY STATEMENT}

The datasets presented in this study can be found in online repositories. The names of the repository/repositories and accession number(s) can be found in the article/Supplementary Material. 


\section{ETHICS STATEMENT}

The studies involving human participants were reviewed and approved by The Institutional Review Boards of The First Affiliated Hospital of Nanchang University. The patients/participants provided their written informed consent to participate in this study. Written informed consent was obtained from the individual(s) for the publication of any potentially identifiable images or data included in this article.

\section{AUTHOR CONTRIBUTIONS}

$\mathrm{CH}$ performed the bioinformatics analysis and wrote the manuscript. HW, CY, and CP collected the samples and contributed to the data collection. XS and WL performed the clinical diagnosis. ZZ designed and supervised the project. All authors contributed to the article and approved the submitted version.

\section{FUNDING}

This research was supported by the National Natural Science Foundation of China (81660101 and 81860106), grants from the Science and Technology Department of Jiangxi Province

\section{REFERENCES}

Chen, S., Zhou, Y., Chen, Y., and Gu, J. (2018). fastp: an ultra-fast all-in-one FASTQ preprocessor. Bioinformatics 34, i884-i890. doi: 10.1093/bioinformatics/bty560

Douglas, G. M., Hansen, R., Jones, C. M. A., Dunn, K. A., Comeau, A. M., Bielawski, J. P., et al. (2018). Multi-omics differentially classify disease state and treatment outcome in pediatric Crohn's disease. Microbiome 6:13. doi: 10.1186/s40168018-0398-3

Eck, A., de Groot, E. F. J., de Meij, T. G. J., Welling, M., Savelkoul, P. H. M., and Budding, A. E. (2017). Robust microbiota-based diagnostics for inflammatory bowel disease. J. Clin. Microbiol. 55, 1720-1732. doi: 10.1128/JCM.00162-17

Edgar, R. C. (2013). UPARSE: highly accurate OTU sequences from microbial amplicon reads. Nat. Methods 10, 996-998. doi: 10.1038/nmeth.2604

Feng, Q., Chen, W. D., and Wang, Y. D. (2018). Gut microbiota: an integral moderator in health and disease. Front. Microbiol. 9:151. doi: 10.3389/fmicb. 2018.00151

He, C., Wang, H., Liao, W. D., Peng, C., Shu, X., Zhu, X., et al. (2019). Characteristics of mucosa-associated gut microbiota during treatment in Crohn's disease. World J. Gastroenterol. 25, 2204-2216. doi: 10.3748/wjg.v25. i1 18.2204

He, Q., Gao, Y., Jie, Z., Yu, X., Laursen, J. M., Xiao, L., et al. (2017). Two distinct metacommunities characterize the gut microbiota in Crohn's disease patients. Gigascience 6, 1-11. doi: 10.1093/gigascience/gix050

He, Y., Zhu, Z., Chen, Y., Chen, F., Wang, Y., Ouyang, C., et al. (2019). Development and validation of a novel diagnostic nomogram to differentiate between intestinal tuberculosis and crohn's disease: a 6-year prospective multicenter study. Am. J. Gastroenterol. 114, 490-499. doi: 10.14309/ajg. 0000000000000064

Hu, Y., Feng, Y., Wu, J., Liu, F., Zhang, Z., Hao, Y., et al. (2019). The gut microbiome signatures discriminate healthy from pulmonary tuberculosis patients. Front. Cell Infect. Microbiol. 9:90. doi: 10.3389/fcimb.2019.00090

Kedia, S., Das, P., Madhusudhan, K. S., Dattagupta, S., Sharma, R., Sahni, P., et al. (2019). Differentiating Crohn's disease from intestinal tuberculosis. World J. Gastroenterol. 25, 418-432. doi: 10.3748/wjg.v25.i4.418
(20192BBG70037 and 20204BCJL23048), and the grant from the Nanchang University (PY201816).

\section{SUPPLEMENTARY MATERIAL}

The Supplementary Material for this article can be found online at: https://www.frontiersin.org/articles/10.3389/fbioe. 2021.673691/full\#supplementary-material

Supplementary Figure 1 | Mucosa-associated gut microbiota biomarkers for classifying patients with intestinal tuberculosis (ITB) from heathy controls $(\mathrm{HC})$. Mean Decrease Gini (A) and Mean Decrease Accuracy (B) analysis showed the top 20 most important genera by the random forest test. (C) The accuracy of candidate biomarkers was verified with cross-validation. The area under curve $(A \cup C)$ value was calculated and receiver operating characteristic curves (ROC) were drawn with five repeats.

Supplementary Figure 2 | Identification of the gut microbial signatureassociated with Crohn's disease (CD). The top 36 most important genera were assessed by the Mean Decrease of Gini (A) andaccuracy (B). (C) The ROC curve of the optimal model containing 3 genera for classifying $\mathrm{CD}$ from healthy controls $(\mathrm{HC})$.

Supplementary Figure 3 | The ITB-diminished genera were significantly increased after 6 months of anti-tuberculosis treatment. One of the ITB patients was followed up and the intestinal ulcer was completely healed after the quadruple regimen (isoniazide, rifampicin, pyrazinamide, ethambutol) for 6 months. The mucosal samples from ileum, ascending colon and descending colon were collected before and after treatment and then the changes of gut microbiota were compared at genus level by Welch's $t$ test.

Kriss, M., Hazleton, K. Z., Nusbacher, N. M., Martin, C. G., and Lozupone, C. A. (2018). Low diversity gut microbiota dysbiosis: drivers, functional implications and recovery. Curr. Opin. Microbiol. 44, 34-40. doi: 10.1016/j.mib.2018.07.003

Langille, M. G., Zaneveld, J., Caporaso, J. G., McDonald, D., Knights, D., Reyes, J. A., et al. (2013). Predictive functional profiling of microbial communities using $16 \mathrm{~S}$ rRNA marker gene sequences. Nat. Biotechnol. 31, 814-821. doi: $10.1038 /$ nbt. 2676

Limsrivilai, J., and Pausawasdi, N. (2021). Intestinal tuberculosis or Crohn's disease: a review of the diagnostic models designed to differentiate between these two gastrointestinal diseases. Intest. Res. 19, 21-32. doi: 10.5217/ir.2019.09142

Liu, P., Wang, Y., Yang, G., Zhang, Q., Meng, L., Xin, Y., et al. (2021). The role of short-chain fatty acids in intestinal barrier function, inflammation, oxidative stress, and colonic carcinogenesis. Pharmacol. Res. 165:105420. doi: 10.1016/j. phrs.2021.105420

Lopetuso, L. R., Petito, V., Graziani, C., Schiavoni, E., Paroni Sterbini, F., Poscia, A., et al. (2018). Gut microbiota in health, diverticular disease, irritable bowel syndrome, and inflammatory bowel diseases: time for microbial marker of gastrointestinal disorders. Dig. Dis. 36, 56-65. doi: 10.1159/000477205

Luo, M., Liu, Y., Wu, P., Luo, D. X., Sun, Q., Zheng, H., et al. (2017). Alternation of gut microbiota in patients with pulmonary tuberculosis. Front. Physiol. 8:822. doi: 10.3389 /fphys.2017.00822

Magoc, T., and Salzberg, S. L. (2011). FLASH: fast length adjustment of short reads to improve genome assemblies. Bioinformatics 27, 2957-2963. doi: 10.1093/ bioinformatics/btr507

Ondov, B. D., Bergman, N. H., and Phillippy, A. M. (2011). Interactive metagenomic visualization in a web browser. BMC Bioinform. 12:385. doi: 10.1186/1471-2105-12-385

Pascal, V., Pozuelo, M., Borruel, N., Casellas, F., Campos, D., Santiago, A., et al. (2017). A microbial signature for Crohn's disease. Gut 66, 813-822. doi: 10. 1136/gutjnl-2016-313235

Ren, Z., Li, A., Jiang, J., Zhou, L., Yu, Z., Lu, H., et al. (2019). Gut microbiome analysis as a tool towards targeted non-invasive biomarkers for early hepatocellular carcinoma. Gut 68, 1014-1023. doi: 10.1136/gutjnl-2017315084 
Thomas, A. M., Manghi, P., Asnicar, F., Pasolli, E., Armanini, F., Zolfo, M., et al. (2019). Metagenomic analysis of colorectal cancer datasets identifies cross-cohort microbial diagnostic signatures and a link with choline degradation. Nat. Med. 25, 667-678. doi: 10.1038/s41591-019-0 405-7

Thukral, A. K. (2017). A review on measurement of Alpha diversity in biology. Agric. Res. J. 54, 1-10. doi: 10.5958/2395-146X.2017.00001.1

Wang, Q., Garrity, G. M., Tiedje, J. M., and Cole, J. R. (2007). Naive Bayesian classifier for rapid assignment of rRNA sequences into the new bacterial taxonomy. Appl. Environ. Microbiol. 73, 5261-5267. doi: 10.1128/AEM.00 062-07

Wang, S., Yang, L., Hu, H., Lv, L., Ji, Z., Zhao, Y., et al. (2021). Characteristic gut microbiota and metabolic changes in patients with pulmonary tuberculosis. Microb. Biotechnol. [Epub ahead of print]. doi: 10.1111/1751-7915.13761

Wang, Y., Gao, X., Ghozlane, A., Hu, H., Li, X., Xiao, Y. T., et al. (2017). Characteristics of fecal microbiota in pediatric crohn's disease and their dynamic changes during infliximab therapy. J. Crohns Colitis 12, 337-346. doi: $10.1093 /$ ecco-jcc/jjx153

Wang, Y., Gao, X., Zhang, X., Xiao, F., Hu, H., Li, X., et al. (2021). Microbial and metabolic features associated with outcome of infliximab therapy in pediatric
Crohn's disease. Gut Microbes 13, 1-18. doi: 10.1080/19490976.2020.186 5708

World Health Organization (2020). Global Tuberculosis Report 2020. Geneva: WHO. Licence: CC BY-NC-SA 3.0 IGO.

Wu, J., Wei, Z., Cheng, P., Qian, C., Xu, F., Yang, Y., et al. (2020). Rhein modulates host purine metabolism in intestine through gut microbiota and ameliorates experimental colitis. Theranostics 10, 10665-10679. doi: 10.7150/thno. 43528

Conflict of Interest: The authors declare that the research was conducted in the absence of any commercial or financial relationships that could be construed as a potential conflict of interest.

Copyright (c) $2021 \mathrm{He}$, Wang, Yu, Peng, Shu, Liao and Zhu. This is an openaccess article distributed under the terms of the Creative Commons Attribution License (CC BY). The use, distribution or reproduction in other forums is permitted, provided the original author(s) and the copyright owner(s) are credited and that the original publication in this journal is cited, in accordance with accepted academic practice. No use, distribution or reproduction is permitted which does not comply with these terms. 